\title{
A model for wave scattering in the marginal ice zone based on a two-dimensional floating-elastic-plate solution
}

\author{
Alison L. KOHOUT, Michael H. MEYLAN \\ Department of Mathematics, University of Auckland, Private Bag 92019, Auckland, New Zealand \\ E-mail: akohout@math.auckland.ac.nz
}

\begin{abstract}
We present a model for wave propagation in the marginal ice zone (MIZ). The model is based on a two-dimensional linear water wave floating-elastic-plate solution in the frequency domain using a matched eigenfunction expansion. The model is fully coherent so the results are dependent on exact geometry, and we show firstly that this dependence can be effectively removed by averaging over random floe lengths. We present a range of predictions from the model which show various curves of transmitted energy as a function of the floe thickness, number of floes and wave period. These curves show that it is possible with this simple model to make predictions about wave intensity with distance into the MIZ. The results predict that the MIZ acts as a low-pass filter and and that the filtering is strongly dependent on floe thickness and number but that it is independent of floe length.
\end{abstract}

\section{INTRODUCTION}

The sea ice that forms in the polar oceans plays a key role in driving the world's oceanic circulatory system and hence the world's climatic system. It is therefore important to understand the processes which influence the extent of this sea ice. Squire and others (1995) and Wadhams (2000) have pointed out evidence to suggest that ocean waves play a major role in the fracturing of ice-covered seas. The analysis of this phenomenon involves many complicated variables, and considerable idealization is required. One aspect which is critical to understand is the wave scattering by the region of broken ice, known as the marginal ice zone (MIZ), which forms at the boundary of open water and frozen seas. This paper presents a simple model for wave propagation through the MIZ.

The MIZ is an interfacial region which forms at the boundary of open and frozen oceans. It consists of a vast field of ice floes and is subject to considerable wave action due to its proximity to the open ocean. Experiments have shown that the wave intensity decays exponentially with distance from the open ocean and that this rate of decay is highly dependent on the wave period (Wadhams and others, 1986, 1988). It has also been shown experimentally that the ice floes can bend significantly (Squire and Martin, 1980), and it is standard to model ice floes as floating elastic plates (Squire and others, 1995).

A number of models for wave propagation in the MIZ have been presented. The most sophisticated were the fully three-dimensional models using a coupling of solutions for individual ice floes with a transport equation (Masson and LeBlond, 1989; Meylan and others, 1997). These models, which were derived separately, have been recently shown to be almost identical (Meylan and Masson, 2006). Another three-dimensional model was developed by Dixon and Squire (2001) based on the coherent potential approximation. We present here a much simpler model based on a two-dimensional solution. While this model is much simpler, it has a number of advantages over the threedimensional models, the most important being that it is much less computationally demanding. We also believe that a simple model is the best place to begin to make comparisons with data and to test and debug the more sophisticated models. We also believe this simpler model will be able to make the kinds of predictions that are required practically, which the more sophisticated models have not been able to do.

The two-dimensional solution is based on a matched eigenfunction expansion. The model is described in detail in Kohout and others (in press) where the solution is carefully analyzed for energy conservation, compared with previous results and compared to a series of experiments which were performed by Sakai and Hanai (2002) using floating plastic sheets in a two-dimensional wave tank. We are therefore confident that the numerical solution presented here is correct.

\section{FORMULATION AND PRELIMINARIES}

We model the MIZ as a set of floating elastic plates occupying the water surface. A small-amplitude wave is incident from the left. We assume the problem is linear and ignore any dissipation The submergence of the plates is considered negligible. We assume that the problem is invariant in the $y$ direction. The set of plates consists of two semi-infinite plates separated by a region consisting of a finite number of plates with variable properties. The left plate edges are at $x=I_{\mu}$, and the right plate edges are at $x=r_{\mu}$ for the $\mu$ th plate. We note that we simulate open water by setting the plate properties, such as the thickness, to a negligibly small value. However, in the results we present here, we consider only contiguous plates except for the plate on the left where we set the thickness sufficiently small that it approximates open water. We also assume that the plate edges are free to move at each boundary. A schematic diagram of the problem is shown in Figure 1. We consider fluid of finite depth because this is required by our solution method. However, we will set the depth to be sufficiently large to be modelled as infinite in the results. The solution method and formulation of this problem is described in detail in Kohout and others (in press).

\subsection{Assumptions and conditions}

We assume that the fluid has constant depth $h$ and occupies the region $-\infty<x, y<\infty$ and $-h<z \leq 0$. In this region 


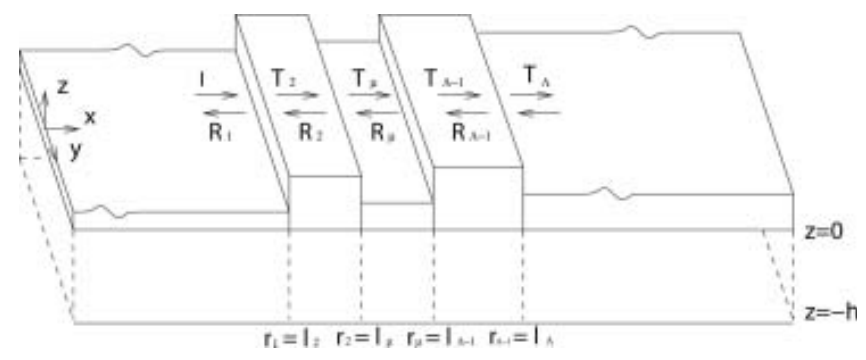

Fig. 1. A schematic diagram showing the set of ice floes and the coordinate systems used in the solution. The three-dimensional region is defined by $-\infty<x, y<\infty$ and $-h<z \leq 0$, where we assume constant depth $h . I$ represents the incident wave. $R_{\mu}$ and $T_{\mu}$ represent the reflection and transmission coefficients of the $\mu$ th plate. $I_{\mu}$ and $r_{\mu}$ represent the left and right edge of the floe $\mu$. There are $\Lambda$ floes.

the fluid flow is irrotational and inviscid so that the fluid velocity can be written and the gradient of a velocity potential $\Phi$ which satisfies Laplace's equation in the fluid region. We consider only incident waves of a single frequency $\omega$ and we assume that these waves also have no variation with respect to $y$. The velocity potential of the wave can therefore be expressed as (Stoker, 1957; Fox and Squire, 1994)

$$
\Phi(x, y, z, t)=\Re\left\{\phi(x, z) \mathrm{e}^{-i \omega t}\right\},
$$

where $\phi$ is a complex-valued potential, $\omega$ is the radian frequency and $\Re$ denotes the real part.

We assume the seabed is impermeable and therefore the velocity component normal to the sea floor vanishes. Hence, the velocity potential at the sea floor satisfies

$$
\frac{\partial \Phi}{\partial z}=0 \quad \text { at } z=-h, \quad \forall x, y .
$$

Using linearity at the free surface, the kinematic condition is

$$
\frac{\partial \eta}{\partial t}=\frac{\partial \Phi}{\partial z} \quad \text { at } z=0, \quad \forall x, y
$$

(Billingham and King, 2000), where $\eta$ is the displacement. We assume the ice floes have uniform mass density $\rho_{\mathrm{i}}$ and thickness $\tau$ (where the thickness of each floe may vary). We assume the amplitude at the free surface is small relative to the wavelength and that the curvature is small and linearity can be applied. We assume the equation of motion for the floe is given by the elastic-plate equation

$$
P=D \partial_{x}^{4} \eta+m \frac{\partial^{2} \eta}{\partial t^{2}} \quad \text { at } z=0, \quad \forall x, y
$$

(Wang and Meylan, 2004), where $P$ is the pressure at the surface, $D$ is the rigidity constant of the plate $(D=$ $Y \tau^{3} /\left[12\left(1-\nu^{2}\right)\right]$, where $Y$ is the Young's modulus and $\nu$ is Poisson's ratio) and $m=\rho_{\mathrm{i}} \tau$.

The dynamic condition at the free surface is given by the linearized Bournoulli's equation

$$
\frac{\partial \Phi}{\partial t}+\frac{P}{\rho}+g \eta=0 \quad \text { at } z=0, \quad \forall x, y
$$

(Stoker, 1957), where $P$ is the pressure at the water surface and $\rho$ is the water density. Equating (4) and (5) gives

$$
D \partial_{x}^{4} \eta+m \frac{\partial^{2} \eta}{\partial t^{2}}+\rho \frac{\partial \Phi}{\partial t}+\rho g \eta=0 \quad \text { at } z=0, \quad \forall x, y .
$$

Additional constraints apply at the edges of the elastic plates (Fox and Squire, 1994). We assume that the plate edges are

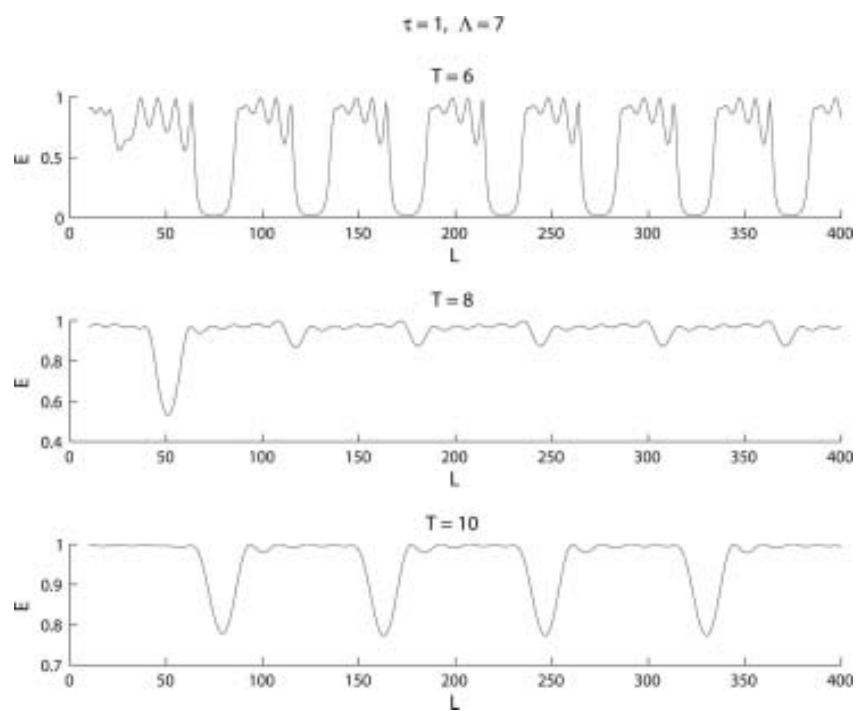

Fig. 2. $E$ vs floe length $L$ for the periods $T$ shown. $\tau=1 \mathrm{~m}$ and $\Lambda=7$. The floes are all of identical length.

free, which implies that the bending moment and the shearing forces at the edges are zero. Therefore the edge boundary conditions can be expressed as

$$
\frac{\partial^{2} \eta}{\partial x^{2}}=0 \quad \text { at } z=0 \quad \text { for } x=I, r, \quad \forall y
$$

and

$$
\frac{\partial^{3} \eta}{\partial x^{3}}=0 \quad \text { at } z=0 \quad \text { for } x=1, r, \quad \forall y
$$

where $I$ and $r$ represent the left and right edge of the plate.

\subsection{Non-dimensionalizing the variables}

It is convenient to reduce the number of constants in the equations by non-dimensionalizing. We non-dimensionalize by scaling the spatial variables by a length parameter $L$, and the time variables by a time parameter $\sqrt{L / g}$. The choice of length parameter $L$ we leave open, but two common values are the water depth $h$ or the characteristic length $L=(D / \rho g)^{1 / 4}$ which have the effect of removing one further variable from the equations. The non-dimensional variables, denoted by an overbar, are

$$
\begin{aligned}
& \bar{x}=\frac{x}{L^{\prime}}, \quad \bar{y}=\frac{y}{L^{\prime}} \quad \bar{z}=\frac{z}{L^{\prime}} \quad \bar{\eta}=\frac{\eta}{L^{\prime}} \\
& \bar{t}=\frac{t}{\sqrt{g / L}} \quad \text { and } \quad \bar{\Phi}=\frac{\Phi}{L \sqrt{L g}} .
\end{aligned}
$$

The boundary condition given by Equation (6) can now be non-dimensionally expressed as

$$
\beta \partial_{\bar{x}}^{4} \bar{\eta}+\gamma \frac{\partial^{2} \bar{\eta}}{\partial \bar{t}^{2}}+\frac{\partial \bar{\Phi}}{\partial \bar{t}}+\bar{\eta}=0 \quad \text { at } z=0, \quad \forall x, y
$$

where $\beta=D /\left(\rho g L^{4}\right)$ is referred to as the stiffness constant and $\gamma=m /(\rho L)$ is referred to as the mass constant. Hereinafter, all equations are expressed non-dimensionally, and for simplicity the overbar will be omitted from the dimensionless variables.

\subsection{Eliminating time}

The time dependence can be eliminated from Equations (2), (9), (7) and (8) using Equation (1) to give us the following 


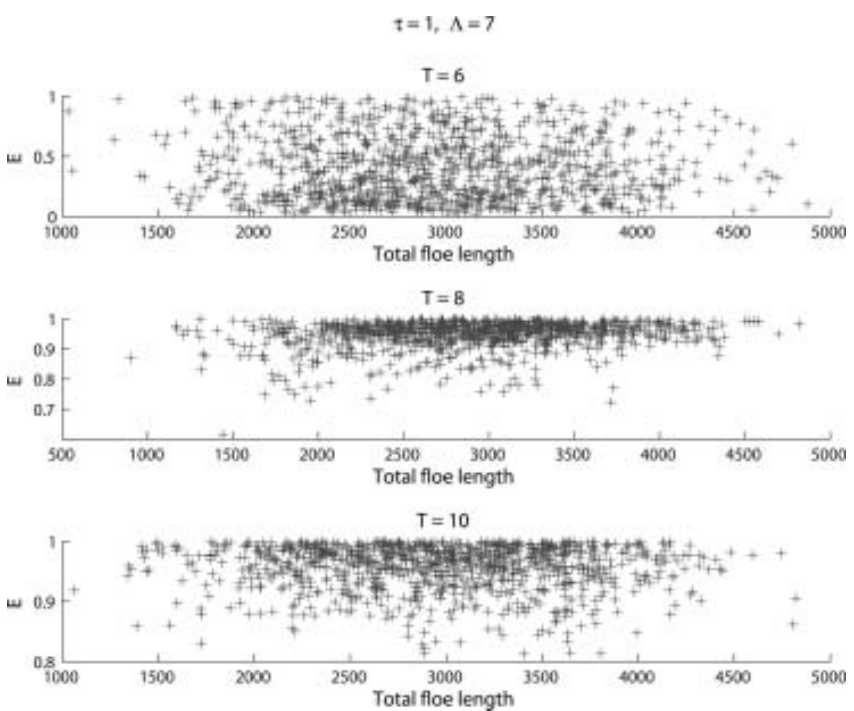

Fig. 3. $E$ vs the total length of the all the finite-length floes for 100 trials where the length of each plate is chosen at random between 10 and $1000 \mathrm{~m}$ for the periods $T$ shown. $\tau=1 \mathrm{~m}$ and $\Lambda=7$.

system of equations throughout the fluid, written entirely in terms of the velocity potential

$$
\begin{array}{rlrl}
\left(\frac{\partial^{2}}{\partial x^{2}}+\frac{\partial^{2}}{\partial z^{2}}\right) \phi & =0 & \text { for }-h<z \leq 0, \quad \forall x \\
\frac{\partial \phi}{\partial z} & =0 \quad \text { at } z=-h, \quad \forall x
\end{array}
$$

and also using Equation (3)

$$
\left(\beta \frac{\partial^{4}}{\partial x^{4}}-\gamma \alpha+1\right) \frac{\partial \phi}{\partial z}-\alpha \phi=0 \quad \text { at } z=0, \quad \forall x
$$

where $\alpha=\omega^{2}$ and

$$
\begin{array}{ll}
\left(\frac{\partial^{3}}{\partial x^{3}}\right) \frac{\partial \phi}{\partial z}=0 & \text { at } z=0 \text { for } x=I, r \\
\left(\frac{\partial^{2}}{\partial x^{2}}\right) \frac{\partial \phi}{\partial z}=0 & \text { at } z=0 \quad \text { for } x=I, r .
\end{array}
$$

\section{METHOD OF SOLUTION}

\subsection{Eigenfunction expansion}

We apply the eigenfunction matching method to solve for the velocity potential. This method has been applied in many situations for linear water wave problems, and the technique is described in Linton and Mclver (2001). The method was developed by Fox and Squire (1994) for the case of the elastic-plate boundary condition.

\subsubsection{Separation of variables}

The potential velocity can be written in terms of an infinite series of separated eigenfunctions of the form $\phi=$ $\mathrm{e}^{k x} \cos [k(z+h)]$, where $k$ satisfies the dispersion equation

$$
k \tan (k h)=-\frac{\alpha}{\beta k^{4}+1-\alpha \gamma} .
$$

The dispersion equation, because it depends on $\beta$ and $\gamma$, has different solutions under floes of different properties. Solving for $k$, the dispersion equation (15) gives one purely imaginary root with positive imaginary part, two complex roots with positive real part (in all physical cases) and an
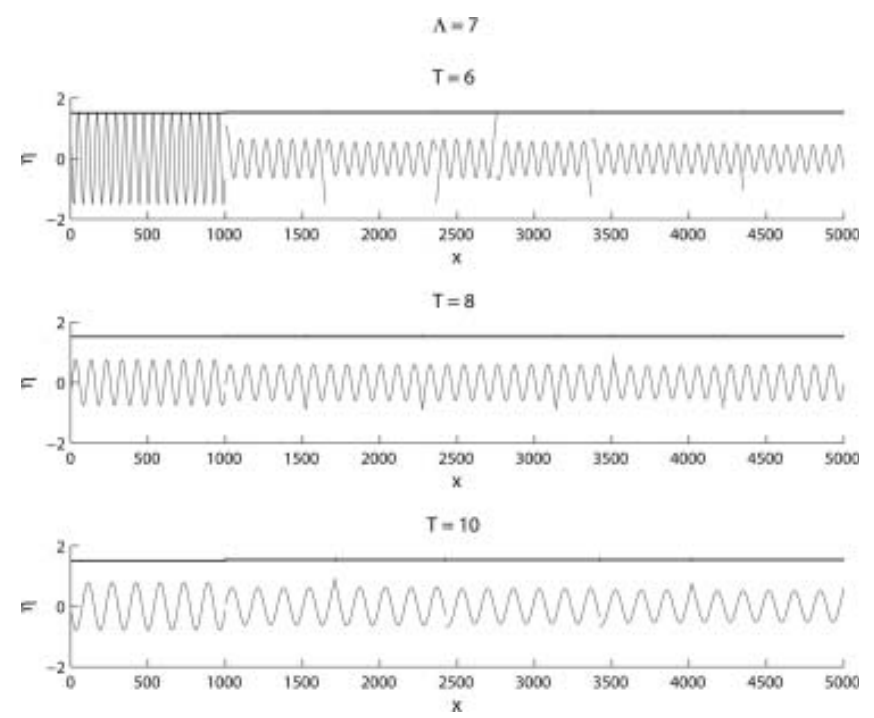

Fig. 4. The displacement vs distance $x$ showing the displacement and floe geometry for three of the realizations used in Figure 3 for the periods $T$ shown. The boxed regions represent each plate.

infinite number of positive real roots which approach $n \pi / h$ as $n$ approaches $\infty$, plus the negative of these roots (Fox and Squire, 1994). The imaginary roots correspond to travelling modes propagating along the $x$ axis. The complex roots correspond to damped travelling modes, and the real roots correspond to the evanescent modes. We denote the solutions of the dispersion equation (15) under the $\mu$ th floe by $k_{\mu}(n)$, where $n=-2,-1$ corresponds to the complex roots with positive real part, $n=0$ corresponds to the imaginary root with positive imaginary part, and $n$ positive corresponds to the real roots with positive real part. This allows us to express the potential as a sum of eigenfunctions under each floe.

\subsection{Expressions for the potential velocity}

The potential $\phi$ can now be expressed as the following sum of eigenfunctions:

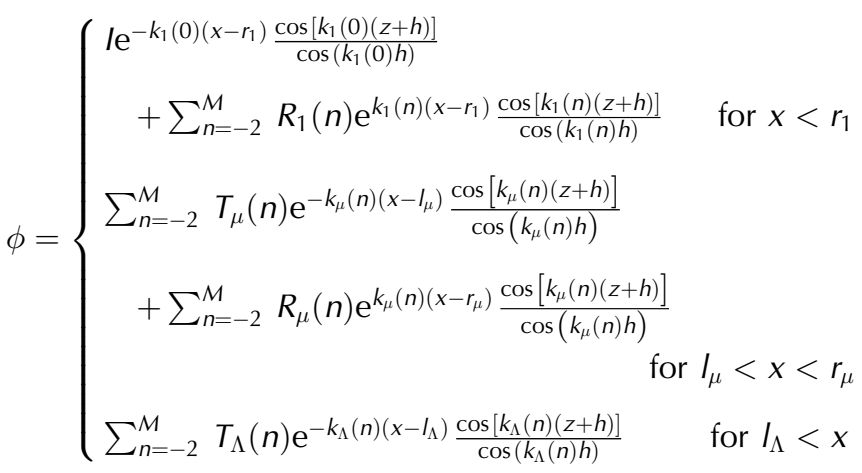

where $\mu$ is the $\mu$ th plate, $\Lambda$ is the last plate, $r_{\mu}$ represents the $x$ coordinate of the right edge of the $\mu$ th plate, $I_{\mu}$ represents the $x$ coordinate of the left edge of the $\mu$ th plate, $R_{\mu}(n)$ represents the reflected potential coefficient of the $n$th mode under the $\mu$ th plate, $T_{\mu}(n)$ represents the transmitted potential coefficient of the $n$th mode under the $\mu$ th plate and $I$ is the incident wave amplitude. Note that we have divided by $\cos (k h)$. This is so the coefficients are normalized by the potential at the top surface rather than at the bottom surface. This means that if the water is deep and the potential at the bottom surface is small, we avoid the problem of having very 

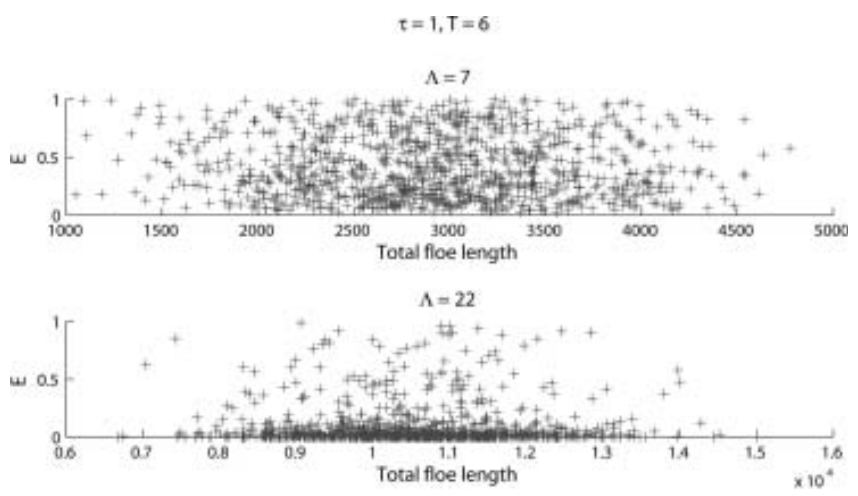

$\Lambda=102$

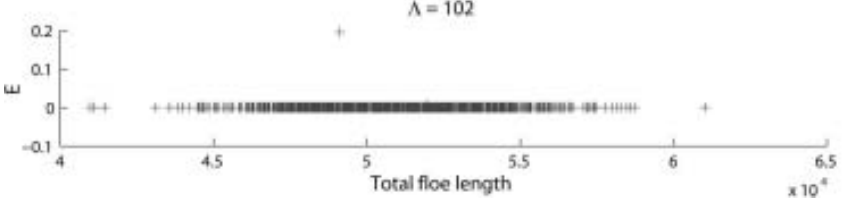

Fig. 5. As Figure 3 except that the period $T=6$ and different values of $\Lambda$ as shown are used.

small numbers for the coefficients in the expansion. We have truncated the expansion at a finite number of modes and we will solve for the coefficents $R_{\mu}(n)$ and $T_{\mu}(n)$ numerically. Finally, we can find the displacement from the potential using Equation (3).

\subsection{Solving via eigenfunction matching}

To solve for the coefficients, we require as many equations as we have unknowns. We impose the condition that the potential and its derivative across each floe boundary are continuous and that the floe satisfies the free-edge conditions. We impose continuity by multiplying the potentials and their derivatives by various orthogonal functions and integrating and imposing the condition that these integrals must be equal. The orthogonal functions we choose are $\cos (m \pi / h)(z+h)$, where $m$ is a natural number. These have the advantage that in the limit of large $n$ the vertical eigenfunctions under each plate take this form. The
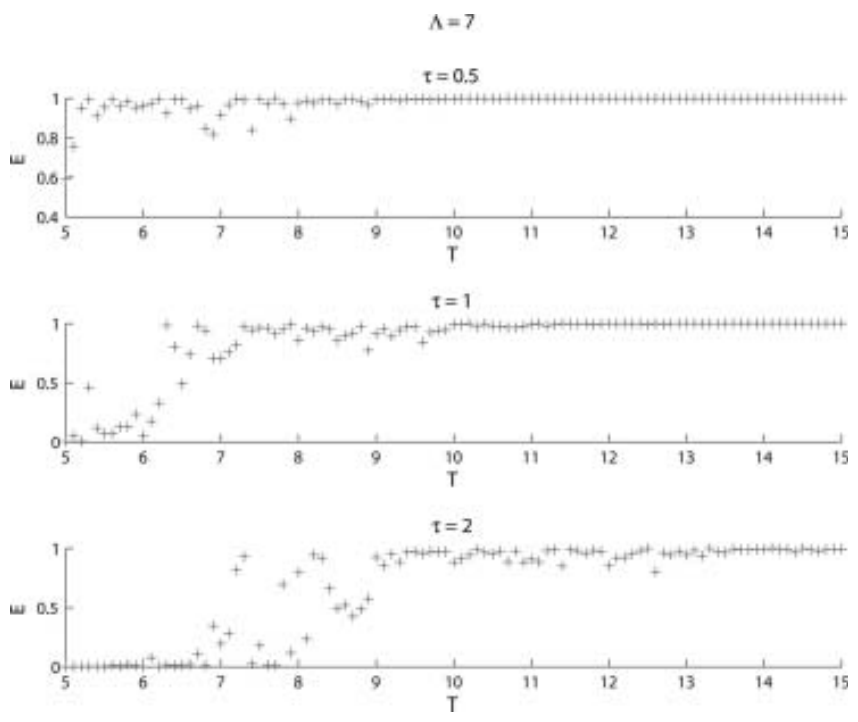

Fig. 6. $E$ vs $T$ for the values of $\tau$ shown for 100 trials. The length of each plate is randomly chosen between 10 and $1000 \mathrm{~m}$.
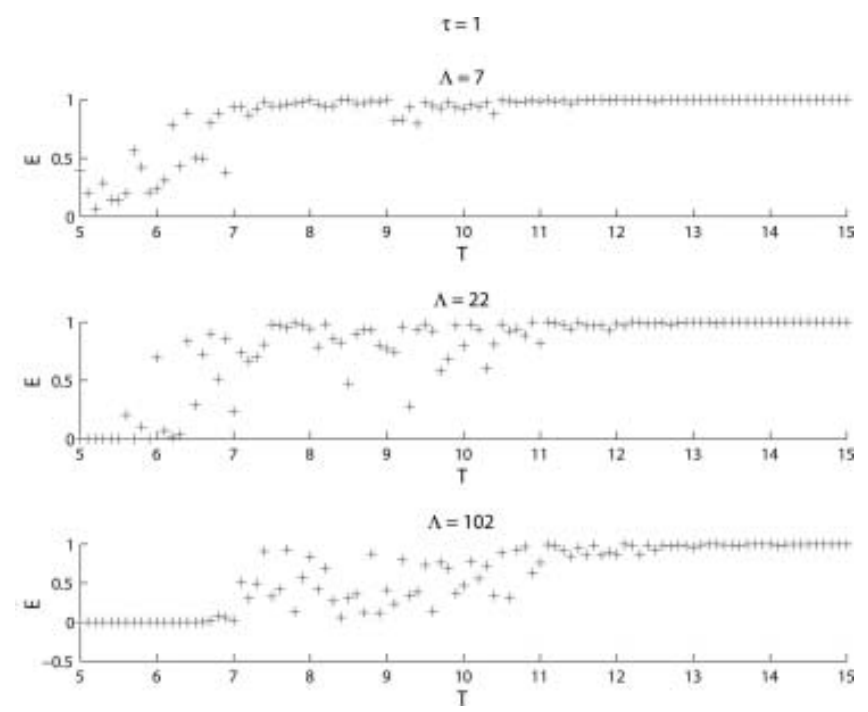

Fig. 7. As Figure 6 except that $\tau=1$ and different values of $\Lambda$ as shown are chosen.

coefficients can now be found through matching the integrals along the water column of the potentials and their derivatives at each adjacent edge:

$$
\begin{aligned}
& \int_{-h}^{0} \phi_{\mu}\left(r_{\mu}, z\right) \cos \frac{m \pi}{h}(z+h) d z \\
& =\int_{-h}^{0} \phi_{\mu+1}\left(I_{\mu+1}, z\right) \cos \frac{m \pi}{h}(z+h) d z, \\
& \int_{-h}^{0} \frac{\partial \phi_{\mu}\left(r_{\mu}, z\right)}{\partial x} \cos \frac{m \pi}{h}(z+h) d z \\
& =\int_{-h}^{0} \frac{\partial \phi_{\mu+1}\left(I_{\mu+1}, z\right)}{\partial x} \cos \frac{m \pi}{h}(z+h) d z,
\end{aligned}
$$

where $m \in[0, M]$ and $\phi_{\mu}$ is the potential under the $\mu$ th
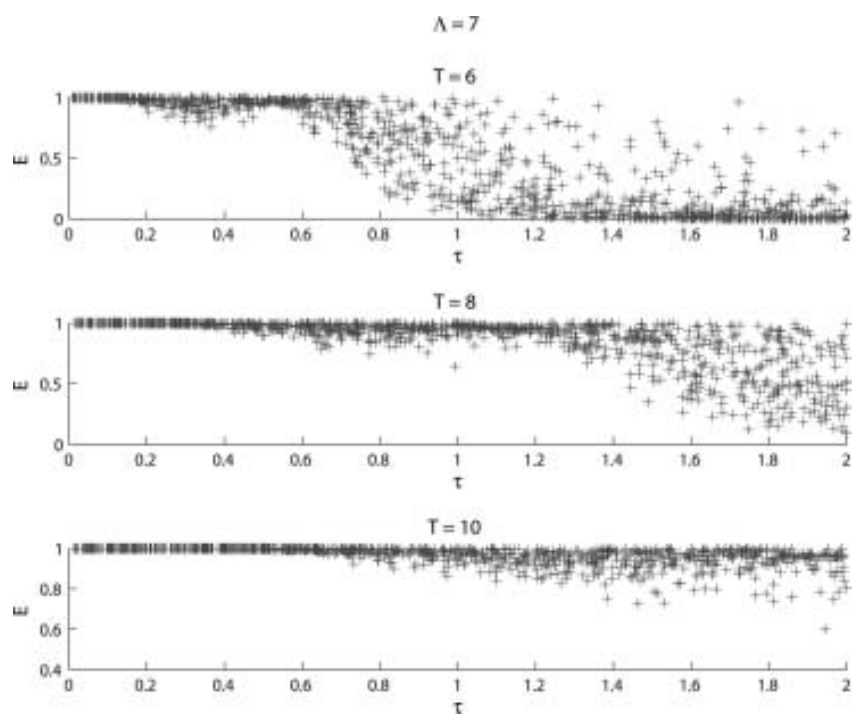

Fig. 8. $E$ vs floe thickness $\tau$ for 100 trials. The length of each plate is chosen at random between 10 and $1000 \mathrm{~m}$. The thickness of the plates is constant for each trial and is chosen at random between 0.01 and $2 \mathrm{~m} . \Lambda=7$. 

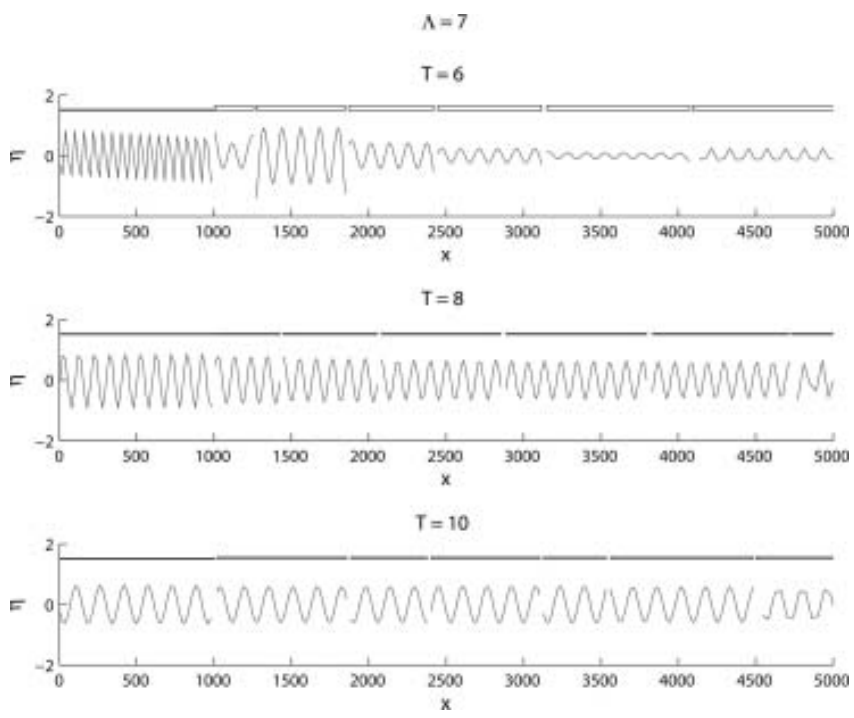

Fig. 9. The displacement vs distance $x$ showing the displacement and floe geometry for three of the realizations used in Figure 8 for the periods $T$ shown. The boxed regions represent each plate.

plate. This gives us four fewer equations than there are unknowns at each plate boundary.

The remaining four equations are given by the two edge conditions at each plate edge:

$$
\begin{aligned}
& \left(\frac{\partial^{3}}{\partial x^{3}}\right) \frac{\partial \phi_{\mu}}{\partial z}=0 \quad \text { for } z=0 \text { and } x=I_{\mu}, r_{\mu} \\
& \left(\frac{\partial^{2}}{\partial x^{2}}\right) \frac{\partial \phi_{\mu}}{\partial z}=0 \quad \text { for } z=0 \text { and } x=I_{\mu}, r_{\mu} .
\end{aligned}
$$

This gives us a system of simultaneous equations which we solve for the unknown coefficients $R_{\mu}$ and $T_{\mu}$.

\section{RESULTS}

We now have a model which can be used to simulate an ocean wave propagating under a region of ice floes. We will set the water depth sufficiently large so that it can be considered infinite and choose the following values
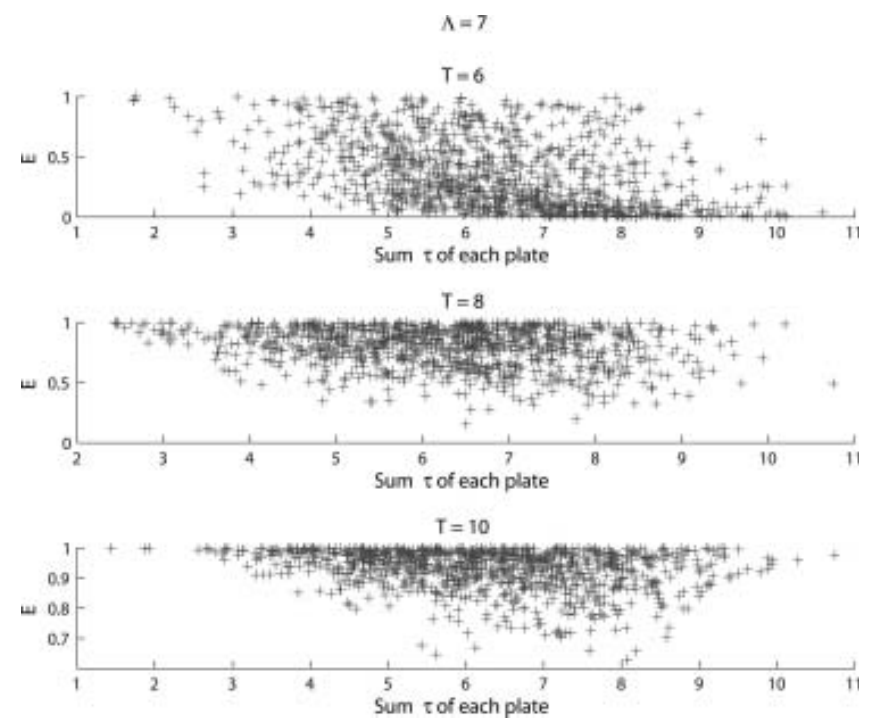

Fig. 11. $E$ vs sum of $\tau$ for each plate, where $\tau$ is chosen at random between 0.01 and $2 \mathrm{~m}$ for each plate for 100 trials for period $T$ shown. $\Lambda=7$.

for the constants: $Y=6 \mathrm{GPa}, \nu=0.3, g=9.8 \mathrm{~m} \mathrm{~s}^{-2}, \rho=$ $1025.0 \mathrm{~kg} \mathrm{~m}^{-3}$ and $\rho_{\mathrm{i}}=922.5 \mathrm{~kg} \mathrm{~m}^{-3}$. We assume that the thickness of the semi-infinite plate on the left is sufficiently small to simulate open water. The semi-infinite plate to the right is a semi-infinite ice floe which is chosen to have the same thickness as the other floes (if the floe thicknesses are different and chosen randomly then the thickness of the semi-infinite floe is also random). A wave is incident from the left hand (open-water side) of unit amplitude and we focus our results on the transmitted energy in the righthand semi-infinite plate which we denote by $E$. We have four free variables: the incident period, $|T|$; the length of each floe, $L$; the thickness of each floe, $\tau$; and the number of floes, $\Lambda$ (note the number of finite floes is $\Lambda-2$ ).

One of the most important features of our model is that it is fully coherent. This means that we will always observe effects of cancellation and addition of waves, no matter how large the ice-floe sizes or number of ice floes. This can be seen in Figure 2 where the transmitted energy in the
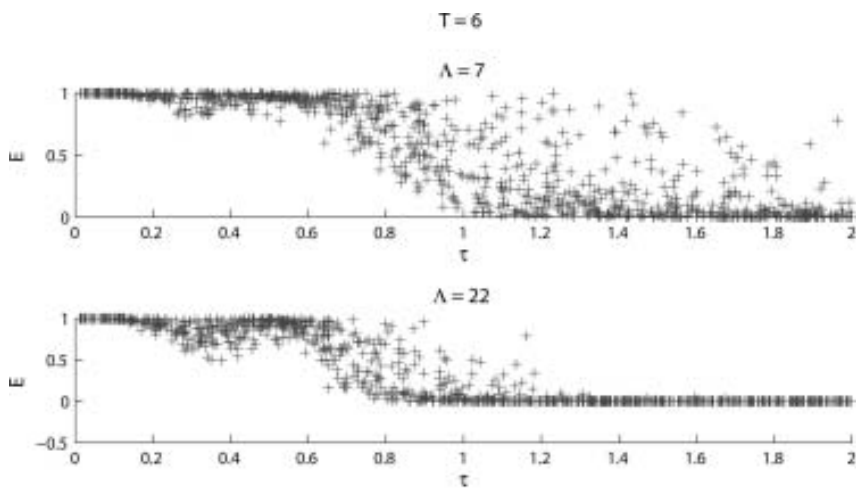

$\Lambda=102$

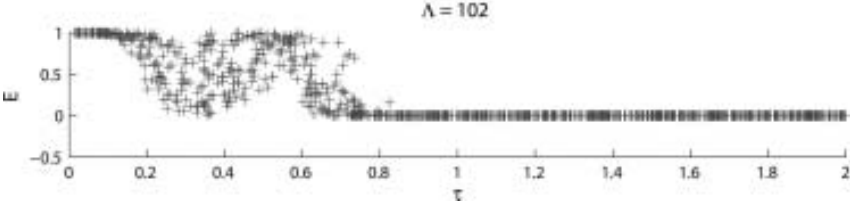

Fig. 10. As Figure 8 except $T=6$ and $\Lambda$ varies as shown.

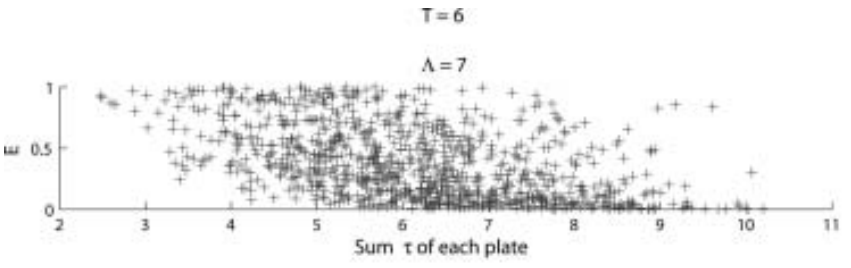

$\Lambda=22$

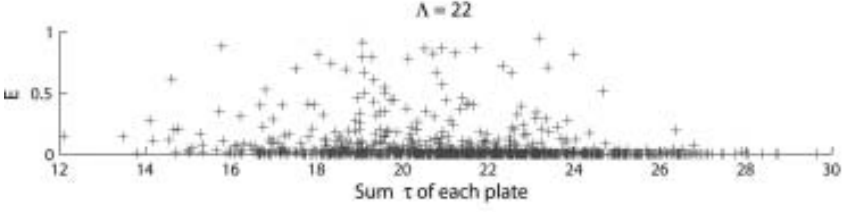

$\Lambda=102$

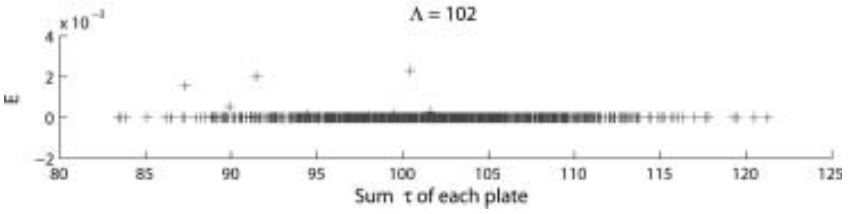

Fig. 12. As Figure 11 except $T=6$ and $\Lambda$ varies as shown. 

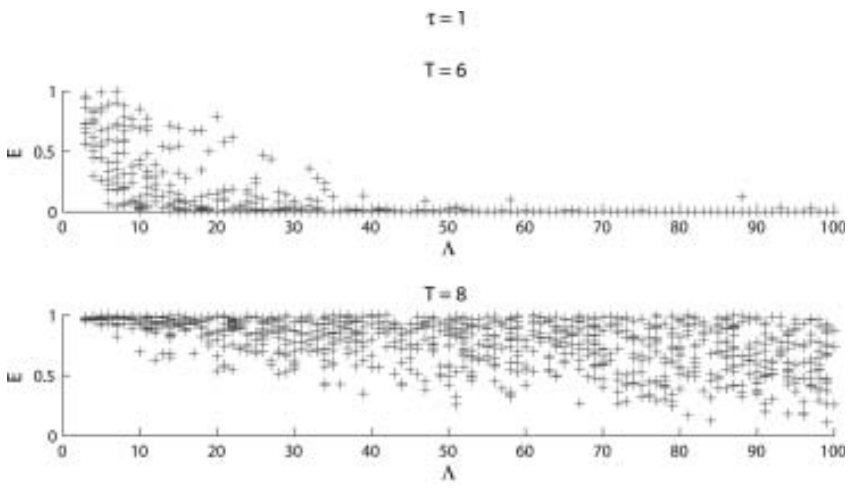

$T=10$

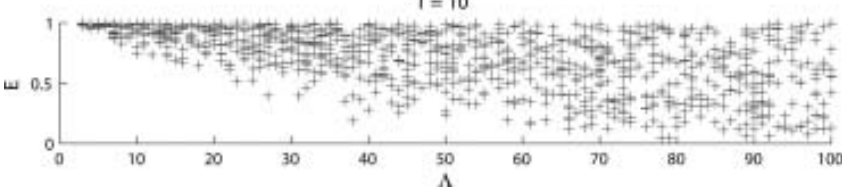

Fig. 13. $E$ vs $\Lambda$ for the periods $T$ shown. $\tau=1 \mathrm{~m}$ and the length of each plate is chosen uniformly at random between 10 and $1000 \mathrm{~m}$.

righthand semi-infinite region, $E$, is shown as a function of floe length for $\Lambda=7$ for the periods of 6,8 and $10 \mathrm{~s}$. To remove this resonating effect, we will always use random floe lengths and will present many different solutions. The floe lengths are selected at random between 10 and $1000 \mathrm{~m}$. The first result we establish is to show that the effect of these random floe lengths can be neglected. Figure 3 shows results for $\Lambda=7$ for the periods shown. Each sample is shown with a plus. It can be seen that there is no correlation between floe length and $E$. Figure 4 shows the displacement for three of the random samples from Figure 3. It is interesting to have some visualization of the solution which is provided by the model for each sample. It can be seen that the model provides much more information than just $E$ since it provides the displacement of each ice floe. This implies that the model could be used to predict other effects such as floe break-up. Figure 5 shows that as we increase the number of ice floes, there is still no correlation with the total floe length. We conclude that the process of setting the floe lengths to be random will be an effective way to remove the coherent effects which were seen in Figure 2, and we will use this in our subsequent work.

Next we examine $E$ in relation to variation in $T$ for fixed floe thickness. Figure 6 shows clear relationships between $E$ and $T$, which depend on the floe thickness; it is also evident that at low periods there is a much greater variability in the value of $E$ than at higher periods. This is to be expected since the greater the reflection, the greater the coherent effects can be. Figure 7 shows that as we increase the number of floes, we get a marked decrease in $E$.

Similar investigations are made into the effect of the floe thickness $\tau$. Figure 8 shows the effect of increasing the floe thickness for different periods and fixed numbers of floes. A strong decease in $E$ can be seen with increasing floe thickness. Figure 9 shows the displacement results for three of the simulations shown in Figure 8. Figure 10 shows the effect of floe thickness for fixed periods and different numbers of floes. This figure also shows the strong dependence of floe thickness on $E$ and that there is a strong decease in $E$ as the number of floes is increased.

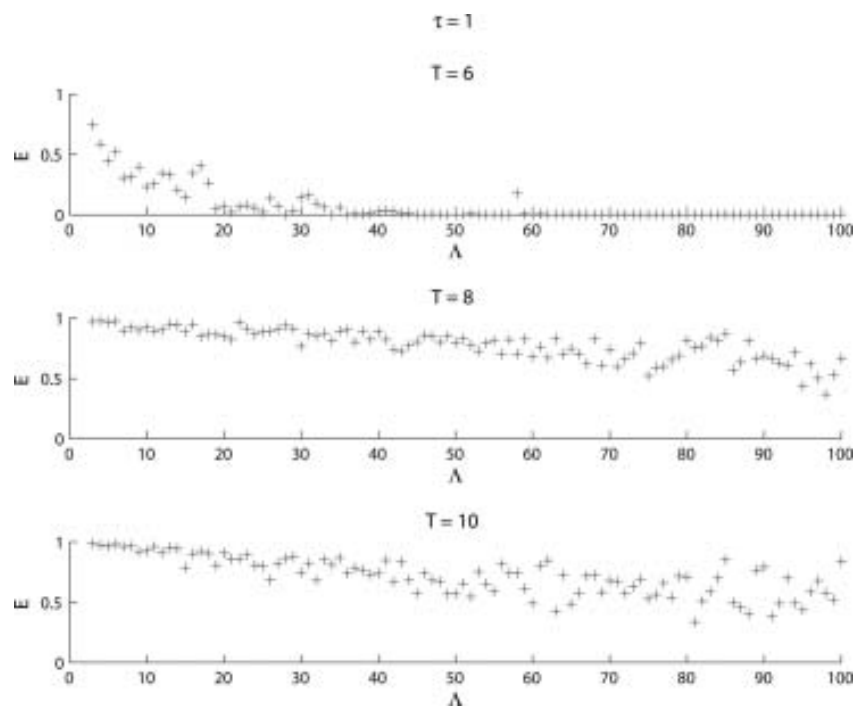

Fig. 14. $E$ vs $\Lambda$ for the periods $T$ shown. $\tau=1 \mathrm{~m}$. $E$ is averaged over five trials where the length of each plate for each trial is chosen uniformly at random between 10 and $1000 \mathrm{~m}$.

Figures 11 and 12 show the effect of the floe thickness on $E$ for random floe thicknesses. Interestingly, considering the strong dependence on $\tau$ in the previous figures, the results for random floe thicknesses do not show the same strong correlation. Figures 11 and 12 show only a slight tendency for a decrease in $E$ as the overall $\tau$ of each floe increases. The reason for the weak correlation is not properly understood and needs to be the subject of further investigation.

Figure 13 shows the effect of increasing the number of floes. We see that $E$ depends strongly on the number of floes and that this dependence is much stronger for small periods. Figure 14 is Figure 13 averaged over five trials. This figure shows more clearly the decay of energy with increasing numbers of floes. It also shows that this decay appears exponential, which agrees with the experimental results of Wadhams and others (1986, 1988).

\section{SUMMARY}

We have presented a model for wave propagation in the MIZ based on a solution for wave propagation under twodimensional floating elastic plates. This model is fully coherent and we showed that this coherence can be removed from the model by averaging over the floe length. We then presented various curves which showed the relationship between transmitted energy and the wave period, floe thickness and number of floes. The results showed that the MIZ acts as a low-pass filter and that the scattering is most strongly dependent on floe thickness and number. The results presented are preliminary results from the model, and future work is planned to establish much more clearly the relationships between the various parameters. We also plan to compare the results with experimental measurements.

\section{REFERENCES}

Billingham, J. and A.C. King. 2000. Wave motion. Cambridge, Cambridge University Press.

Dixon, T.W. and V.A. Squire. 2001. Energy transport in a marginal ice zone. J. Geophys. Res., 106(C9), 19,917-19,928. 
Fox, C. and V.A. Squire. 1994. On the oblique reflexion and transmission of ocean waves at shore fast sea ice. Philos. Trans. R. Soc. London, Ser. A, 347(1682), 185-218.

Kohout, A., M.H. Meylan, S. Sakai, K. Hanai and P. Leman. In press. Linear water wave propagation through multiple floating elastic plates of variable properties. J. Fluid Struct.

Linton, C.M. and P. Mclver. 2001. Handbook of mathematical techniques for wave/structure interactions. Boca Raton, FL, etc., Chapman \& Hall/CRC.

Masson, D. and P.H. LeBlond. 1989. Spectral evolution of windgenerated surface gravity waves in a dispersed ice field. J. Fluid Mech., 202, 43-81.

Meylan, M.H. and D. Masson. 2006. A linear Boltzmann equation to model wave scattering in the marginal ice zone. Ocean Model., 11(3-4), 417-427.

Meylan, M., V.A. Squire and C. Fox. 1997. Towards realism in modelling ocean wave behavior in marginal ice zones. J. Geophys. Res., 102(C10), 22,981-22,991.

Sakai, S. and K. Hanai. 2002. Empirical formula of dispersion relation of waves in sea ice. In Ice in the Environment: Proceedings of the 16th IAHR International Symposium on Ice, Dunedin, New Zealand, 2-6 December 2002, Vol. 2. Dunedin, International Association of Hydraulic Engineering and Research, 327-335.
Squire, V.A. and S. Martin. 1980. A field study of the physical properties, response to swell, and subsequent fracture of a single ice floe in the winter Bering Sea. Seattle, WA, University of Washington. Department of Atmospheric Sciences and Oceanography. (U.S. Office of Naval Research Scientific Report 18.)

Squire, V.A., J.P. Dugan, P. Wadhams, P.J. Rottier and A.K. Liu. 1995. Of ocean waves and sea ice. Annu. Rev. Fluid Mech., 27, 115-168.

Stoker, J.J. 1957. Water waves: the mathematical theory with applications. New York, Wiley Interscience.

Wadhams, P. 2000. Ice in the ocean. Amsterdam, etc., Gordon and Breach Science Publishers.

Wadhams, P., V.A. Squire, J.A. Ewing and R.W. Pascal. 1986. The effect of the marginal ice zone on the directional wave spectrum of the ocean. J. Phys. Oceanogr., 16(2), 358-376.

Wadhams, P., V.A. Squire, D.J. Goodman, A.M. Cowan and S.C. Moore. 1988. The attenuation rates of ocean waves in the marginal ice zone. J. Geophys. Res., 93(C6), 6799-6818.

Wang, C.D. and M.H. Meylan. 2004. A higher-order-coupled boundary element and finite element method for the wave forcing of a floating elastic plate. J. Fluid Struct., 19(4), $557-572$. 